\title{
Estimating potential savings in cancer deaths by eliminating regional and social class variation in cancer survival in the Nordic countries
}

\author{
Paul W Dickman, Robert W Gibberd, Timo Hakulinen
}

\begin{abstract}
Study objectives-To examine equity in the health care system with regard to cancer patient care by estimating the level of systematic regional variation in cancer survival in the Nordic countries. Specifically, those cancer sites which exhibit high levels of systematic regional variation in survival and hence inequity were identified. Estimating the reduction in cancer deaths which could be achieved by eliminating this variation so that everyone receives effective care will provide a readily interpretable measure of the amount of systematic regional variation. A comprehensive analysis of regional variation in survival has not previously been conducted so appropriate statistical methodology must be developed.
\end{abstract}

Setting and participants-All those aged 0-90 years who had been diagnosed with at least one of 12 common malignant neoplasms between 1977 and 1992 in Denmark, Finland, Norway, and Sweden.

Design-A separate analysis was conducted for each country. Regression models for the relative survival ratio were used to estimate the relative risk of excess mortality attributable to cancer in each region after correcting for age and sex. An estimate of the amount of systematic regional variation in survival was obtained by subtracting the estimated expected random variation from the observed regional variation. An estimate was then made of the potential reduction in the number of cancer deaths for 2008-12 if regional variation in survival were eliminated so that everyone received the same level of effective care.

Main results-Between 2008 and 2012, an estimated $2.5 \%$ of deaths from cancers in the 12 sites studied could be prevented by eliminating regional variation in survival. The percentage of potentially avoidable deaths did not depend on country or sex but it did depend on cancer site. There was no relationship between the level of regional variation in a given country and the level of survival. The cancer sites for which the greatest percentage savings could be achieved were melanoma (11\%) and cervix uteri (6\%). The sites for which the highest number of deaths could be prevented were prostate, colon, melanoma, and breast.
Conclusions-This methodology showed a small amount of systematic regional variation in cancer survival in the Nordic countries. The cancer sites with high levels of regional variation identified are potential targets for cancer control programmes.

\section{( $($ Epidemiol Community Health 1997;51:289-298)}

Previous components of the Nordic collaborative project "Cancer in the Nordic countries in the years 1990, 2000 and 2010" (CiN) have predicted cancer incidence and mortality up to the year $2010 .{ }^{12}$ In the current project we examine regional differences in survival within Denmark, Finland, Norway, and Sweden and estimate the potential saving in mortality which could be achieved by eliminating systematic regional variation in cancer patient survival such that everyone receives effective care.

The analysis of geographic variation is a popular methodology in health services research although the statistical methodology for such analyses has not been fully developed. ${ }^{34}$ Most methods have been developed for the study of regional variation in rates, and have been applied to hospital admission rates, surgery rates, and in the field of cancer epidemiology, incidence, and mortality rates. ${ }^{5-9}$ The most appropriate methods for our purposes have focused on answering the question: Is there more variation than can be explained by random factors and, if so, what is the size of the systematic variation? ${ }^{5}$ We have developed a similar methodology for studying regional variation in survival, although the methodology is complicated by the fact that survival is mathematically more difficult to estimate than incidence or mortality rates.

After estimating the level of systematic regional variation in cancer survival, the potential reduction in cancer mortality which could be achieved in the years 2008-12 by eliminating this variation is estimated. Any overall reduction in mortality is brought about by improving the survival in those regions with low survival such that survival rates in all regions are equivalent to what would be expected if everyone received the same level of effective care. This reduction in mortality is additional to any reduction (or increase) in mortality due to trends in survival brought about by other factors.

The Nordic countries consist of Denmark, Finland, Iceland, Norway, and Sweden. The population of Iceland (250 000) is too small to 
facilitate a regional analysis so this study is restricted to the other four countries. We have taken the liberty of referring to these four countries as the Nordic countries.

Cancer care refers to that component of cancer control pertinent to the period following the onset of cancer. It consists of three main components-diagnosis, treatment, and rehabilitation. Cancer control is a more general term which also encompasses, among other areas, primary prevention. Cancer care is defined as being effective if it accomplishes its purpose, which is achieving the best possible patient survival, given the existing state of knowledge. The survival time for a cancer patient is defined as the elapsed time between diagnosis and death and is the principal measure of the effectiveness of cancer care. Effective cancer care implies effective diagnosis, effective treatment, and effective rehabilitation (in terms of survival time).

Equity has been regarded as one of the key indicators of the quality of health care, ${ }^{10}$ although not everyone agrees on the definitions of equity and health. ${ }^{11-15}$ Equity has been equated to access to health care, the allocation of resources, the use of health services, and to per capita expenditure on health care. An alternative to these resource based measures of equity is to use an outcome based measure of equity, namely patient survival. ${ }^{16}$ Using this definition, inequity stems from differences in the effectiveness of cancer care, where effectiveness is measured by patient survival.

Outcome based equity usually implies resource based inequity since population subgroups will require disparate expenditure of resources in order to achieve the same outcome. In many situations, outcome based equity is an unreachable goal since not everyone can be guaranteed the same level of good health (eg those with congenital defects). It is for this reason that most definitions of equity are based on the principle of equality of access rather than equality of "health". An outcome based measure, however, is appropriate for cancer care since we can control for general mortality and it is not unreasonable to aim for nationwide equality of survival (within age/sex/site groups) following a diagnosis of cancer. It still may not be possible to achieve complete equity of cancer survival within each age/sex/site group since further subgroups can usually be identified (based on, for example, smoking habits or ethnic origin) which are subject to dissimilar general mortality. Nevertheless, a target of nationwide equality of survival (within age/sex/ site groups) following a diagnosis of cancer provides a useful and realistic baseline from which to assess equity of cancer care.

If there is a state of equity, there should be no systematic differences in cancer survival between patient groups (within each age/sex/ site) defined by social class or place of residence. Inferences can therefore be made about equity in a health care system by comparing cancer survival across geographic regions and social classes. Karjalainen ${ }^{1117}$ lists three factors which may account for observed differences in cancer patient survival: inequity, confounding variables, and random variation. The aim of this analysis is to estimate the amount of systematic variation in regional survival by controlling for confounding variables and estimating the level of random variation. The amount of systematic variation in regional survival will provide a measure of the level of inequity in the health care system for cancer patients and can highlight areas where there is potential for cancer control programmes to be effective.

\section{Methods}

Case specific data were obtained from the central cancer registry in each country for cases diagnosed from 1977 until the most recent year available, which was 1991 for Denmark and Sweden and 1992 for Finland and Norway. Cases were followed up for deaths to the end of 1991 in Denmark and Sweden, 1992 in Norway, and 1993 in Finland. Notification of new cancer cases is compulsory in each country and the data maintained by each of these population based, nationwide registries is considered to be of the highest quality. ${ }^{2}$ Collaborative projects and annual meetings of the Nordic cancer registries ensure that data collection and follow up procedures are comparable between the Nordic countries.

Each cancer registry also supplied general population mortality rates, obtained from their central statistical offices, which were required for the analysis of relative survival. For Finland, population mortality rates were also provided for each of 12 counties and each of six social classes in addition to the nationwide rates. The Oncologic Centre in Umeå, Sweden, provided standardised mortality ratios for each county, sex, and age group which enabled calculation of region specific population mortality rates for Sweden. Incidence and mortality rates were obtained from computer disks accompanying the $\mathrm{CiN} 1 \mathrm{a}^{1}$ and $1 \mathrm{~b}^{2}$ publications, as were age/sex population figures for 1983-87 and predictions for 2008-12. For Finland, information on social class was available for each case and was used to analyse social class differences in survival in addition to the analysis of regional differences. Social class information was not available for the present study for any of the other three countries. Region specific mortality data were not available for Norway and Denmark.

\section{SITES}

The 12 cancer sites, their corresponding $I C D$ 7 codes, and the number of incident cases for each country are given in table 1 . The sites were chosen so as to obtain a range of survival rates and potential for successful diagnosis and treatment. Sites with a high incidence were primarily chosen in order to reliably estimate the region specific survival. The 12 chosen sites account for $63 \%$ of the total number of cancer deaths during 2008-12 predicted by $\mathrm{CiN} 1 \mathrm{~b}^{2}$ 
Table 1 Number of cases included in the analysis for each cancer site

\begin{tabular}{llrrrrr}
\hline ICD-7 code & Site & Denmark & Finland & Norway & Sweden & \multicolumn{1}{c}{ All } \\
\hline 151 & Stomach & 11261 & 18036 & 14394 & 23278 & 66969 \\
153 & Colon & 27590 & 13523 & 24007 & 39118 & 104238 \\
154 & Rectum & 15924 & 9969 & 12959 & 23485 & 62337 \\
161 & Larynx (male) & 2897 & 1926 & 1777 & 2765 & 9365 \\
$162-163$ & Lung & 41371 & 33899 & 21602 & 34891 & 131763 \\
170 & Breast (female) & 39224 & 32545 & 27735 & 65857 & 165361 \\
171 & Cervix uteri & 8511 & 2746 & 5875 & 7946 & 25078 \\
172 & Corpus uteri & 9234 & 7692 & 6262 & 13859 & 37047 \\
177 & Prostate & 18916 & 18373 & 26771 & 60156 & 124216 \\
180 & Kidney & 8279 & 7799 & 7297 & 15274 & 38649 \\
181 & Urinary bladder & 20430 & 8870 & 14222 & 24084 & 67606 \\
190 & Melanoma of skin & 8689 & 6342 & 10492 & 16357 & 41880 \\
& Total & 212326 & 161720 & 173393 & 327070 & 874509 \\
\hline
\end{tabular}

Table 2 Number of cases excluded from the analysis

\begin{tabular}{lccccccc}
\hline Country & $\begin{array}{l}\text { No } \\
\text { region }\end{array}$ & $\begin{array}{l}\text { Age } \\
>90 y\end{array}$ & Autopsy & DCO* & $\begin{array}{l}\text { Previous } \\
\text { tumourt }\end{array}$ & $\begin{array}{l}\text { Total } \\
\text { excluded }\end{array}$ & $\begin{array}{l}\% \text { of } \\
\text { total }\end{array}$ \\
\hline Denmark & 8 & 2506 & 11740 & 2400 & 887 & 17541 & 7.6 \\
Finland & 2 & 1285 & 4201 & 1092 & 824 & 7404 & 4.4 \\
Norway & 0 & 2082 & 1709 & 1334 & 2081 & 7206 & 4.0 \\
Sweden & 11 & 4375 & 18491 & $0 \neq$ & 3939 & 26816 & 7.6 \\
\hline
\end{tabular}

* DCO: information available from death certificate only

Excluded because of a previous primary tumour in the same site.

$\ddagger$ DCO cases already excluded by the cancer registry.

REGIONS

The regions had to be large enough to allow accurate estimation of survival, yet small enough to reflect regional differences. Regions were defined using pre-existing administrative borders and consisted of 22 health care districts in Finland, 24 counties (län) in Sweden, 16 counties (amter) in Denmark, and 19 counties (fylker) in Norway. The average regional populations were $226,359,321$, and 226 thousand, respectively. It could be argued that it is scientifically more valid to define the regions such that they are homogeneous with respect to demographic or geographic characteristics which are of direct interest (such as socioeconomic status or some measure of access to health care). However, any future action which may be taken to reduce regional variation will require action by the regional health authorities, thus making it perhaps more appropriate to use these administratively defined regions.

\section{EXCLUSIONS}

Some cases were excluded from the analysis in order to obtain a more uniform material, primarily across regions within each country, but also across the different countries (table 2). Reasons for exclusion were region of residence information not available, age at diagnosis greater than 90 years, diagnosis first made at autopsy, and information available from death certificate only (DCO). The survival time will be zero when the diagnosis is first made at autopsy or on the basis of death certificate only.

When a registry receives a death notification for a patient (or tumour) which was not previously known to the registry, the registry contacts the appropriate hospital or physician in an attempt to ascertain the true date of diagnosis. Sometimes it is not possible to ascertain the true date of diagnosis and it is these cases which were excluded. When multiple primary tumours were registered in the same site for the same person, only information relating to the first tumour was included in the analysis. Multiple primary tumours in different sites were included independently in each site, provided other selection criteria were met.

For the Danish material, 5473 cases were excluded due to the diagnosis being first made at autopsy. An additional 6267 cases where only information from the autopsy report was available were excluded and are also reported in this category. The true diagnosis date for these 6267 cases may have been earlier but no additional information is available. The regional distribution of the excluded cases from each country were examined for systematic patterns which may introduce biases. The analysis was also repeated without excluding any cases as a means of testing the sensitivity of the results to the exclusions.

\section{Statistical methods}

The methodology developed for the current study of regional variation in survival can be described in three broad steps:

- Step 1 -estimate survival for each region by modelling relative survival as a function of relevant predictor variables (age, sex, and region) for each cancer site

- Step 2-estimate the amount of systematic (as opposed to random) regional variation in survival

- Step 3-estimate the reduction in mortality which could be achieved if this variation were eliminated such that everyone receives the same level of effective care

Statistical models for relative survival analysis (step 1) are reasonably well developed, although little research has been conducted on methods for studying regional variation in survival (step 2). We have developed methods for examining regional variation in survival which have been applied in the current study.

\section{STEP 1: MODEL FITTING}

The analysis of survival from population based cancer registries with long term follow up is often performed using relative survival analysis. ${ }^{18-20}$ We used the regression model developed by Hakulinen and Tenkanen, ${ }^{21}$ which models the total mortality (number of deaths divided by the person-time at risk) for persons diagnosed with cancer, $\mu$, as the sum of the known baseline mortality, $\mu^{*}$, and the excess mortality due to a diagnosis of cancer, $v$. That is,

$$
\mu_{r i j}=\mu_{(r) i s}^{*}+v_{r i s j}
$$

The subscripts indicate that the excess mortality of the patients (and hence their total mortality) depends on region $(r)$, age $(i)$, sex $(s)$, and follow up interval $(j)$. The baseline mortality depends on age, sex, and possibly region. The model was fitted individually to each of the 12 cancer sites in each of the four countries using the predictor variables region, 
age, sex, and an age by follow up interaction term (for the first five years of follow up only). The age variable contained three levels and was defined separately for each site. Each region was represented by a dummy variable in the regression model and an estimate of the relative risk of excess mortality due to cancer was obtained for each region. The age by follow up interaction term was required in order to obtain an acceptable fit to the data and indicates that the age specific hazards are non-proportional for the first five years of follow up.

The excess mortality component is assumed to be a multiplicative function of the covariates, such that

$$
v_{\text {risj }}=\exp \left(\beta_{r}^{\text {reg }}+\beta_{i}^{\text {age }}+\beta_{j}^{\text {sex }}+\beta_{i j}^{\text {ageffu }}\right)
$$

The individual model parameters (the $\beta$ 's) are interpreted as log relative risks of excess mortality due to cancer. As such, the relative risk of excess mortality due to cancer for residents of region $r$ is given by $R R_{r}=\exp \left(\beta_{r}\right)$, where the superscript reg has been omitted for simplicity. Mortality after $t$ years of follow up, $\mu(t)$, is directly related to the survival rate $S(t)$ through $S(t)=\exp [-\mu(t)]$. The annual (interval specific) relative survival ratio for follow up interval $j$ (and other covariates at levels $r, i$, and $s$ ) is given by

$$
r_{\text {risj }}=\exp \left(-v_{\text {risj }}\right)
$$

Estimated region specific relative risks for Sweden, given by $\mathrm{RR}_{r}=\exp \left(\hat{\beta}_{r}\right)$, where $\hat{\beta}_{r}$ is the estimate for $\beta_{r}$, are presented in figure 1 as an example. A relative risk of 1.4 for a given region indicates that the risk of death due to the cancer in question is 1.4 times higher in that region than it is according to the average of all region specific excess mortalities. Each relative risk represents the ratio of the risks of cancer mortality after correcting for mortality due to other causes.

EXPECTED SURVIVAL

The relative survival ratio (also called the relative survival rate) at time $t$ is the ratio of the observed survival of the cases to the expected survival for a comparable group in the general population. That is, $r(t)=S(t) / S^{*}(t)$, where $S(t)=\exp [-\mu(t)]$ and $S^{*}(t)=\exp \left[-\mu^{*}(t)\right]$. Hence for the current model (1), we have $r(t)=$ $\exp \left[-\mu(t)+\mu^{*}(t)\right]=\exp [-v(t)]$, where $r(t)$, $\mu(t), \mu^{*}(t)$, and $v(t)$ are assumed to be constant for all values of $t$ within each annual follow up interval $j$ and subscripts for the other covariates are omitted.

When conducting a relative survival analysis, it is standard practice to estimate the expected survival rate $S^{*}(t)$ using age/sex specific mortality tables for the entire (nationwide) population. We would not expect the baseline mortality to be identical in all regions, so using nationwide life tables to estimate the expected survival will create bias. This bias is not of great concern when analysing nationwide data but may be important when we wish to estimate survival in individual regions or social class groups.

In order to study the effect of this bias, we calculated expected survival for the Finnish and Swedish material using region specific population life tables. In addition, we also estimated the expected survival for Finland using

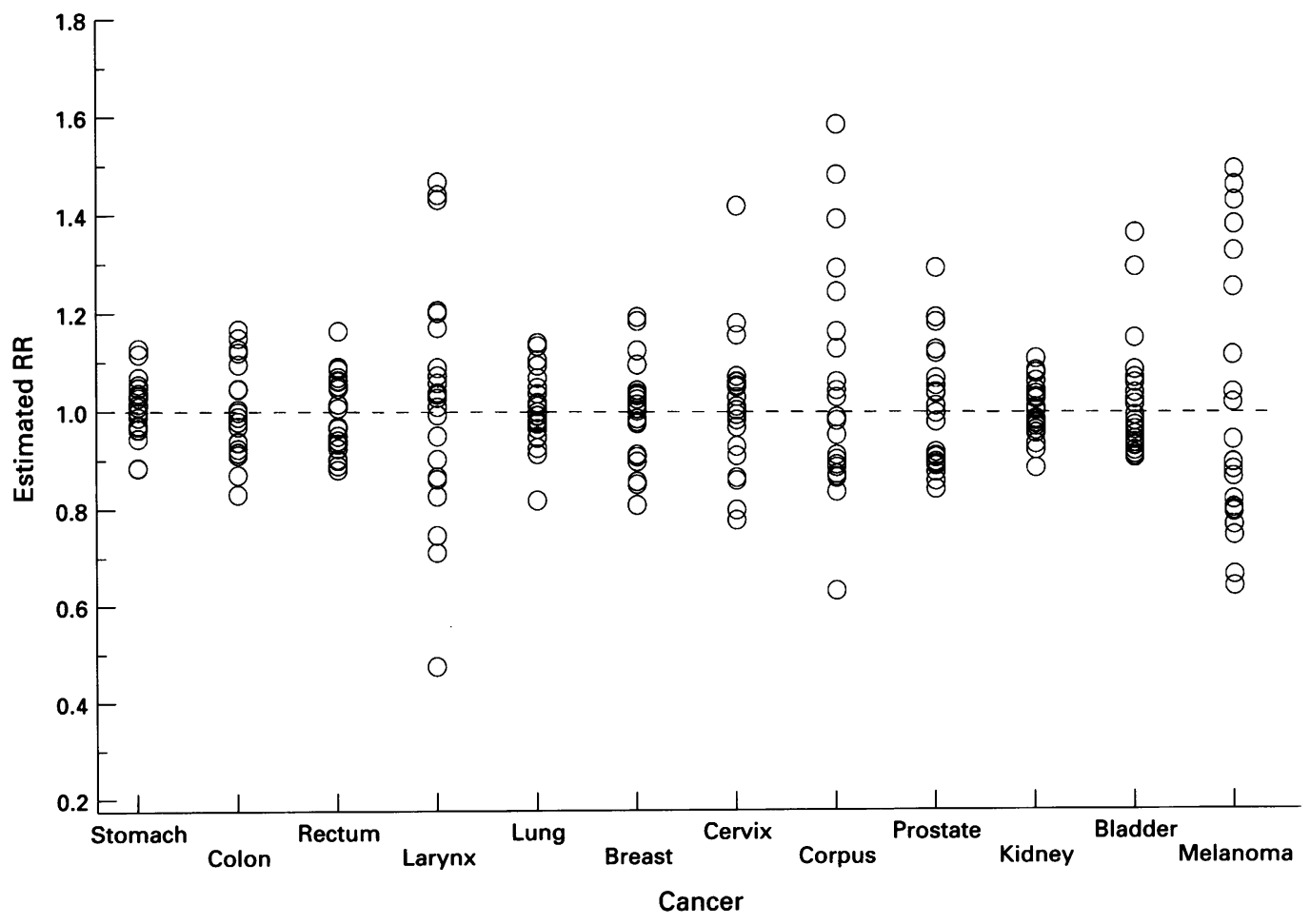

Figure 1 Region specific estimates of the relative risks of excess mortality due to cancer in relation to site for 24 regions in Sweden, 1977-91. Estimates are from the regression model controlling for age, sex follow up, and an age* follow up interaction. 
social class specific life tables. The differences between these three methods of estimating the expected survival and the effect on the final results were studied. For the Norwegian and Danish material, we had no option other than using nationwide life tables to calculate expected survival. The additional analyses for Finland and Sweden were used to assess the magnitude of the bias due to using nationwide life tables.

STEP 2: ESTIMATING THE SYSTEMATIC COMPONENT OF REGIONAL VARIATION The estimates presented in figure 1 are unbiased estimates of the effect of living in each region and, like all estimates, are subject to random variation. For any given cancer site in figure 1 , it is possible that all of the observed variation among the region specific estimates is due to random variation, or it may be due to a combination of random variation and systematic differences in relative survival between the regions. It is possible to estimate the magnitude of the expected random variation, which depends on the number of cases and the survival rate. We developed an estimator $V$ to estimate the magnitude of the systematic regional variation in survival by subtracting the estimated random variation from the observed variation in the estimates $b_{1}, \ldots, b_{r}$ An appendix contains mathematical details of $V$, which is an estimator for

$$
\frac{1}{p-1} \sum_{r=1}^{p}\left(\beta_{r}-\bar{\beta}\right)^{2}
$$

the variance of the ensemble of true regional effects. The value of $V$ will be zero (or negative) if no systematic regional variation in survival exists and will increase in magnitude as the level of systematic variation increases.

As was noted earlier, the estimated region specific relative risks presented in figure 1 are subject to both systematic and random variation. It is possible to calculate new estimates, so called second stage estimates (or shrinkage estimates), which differ solely due to systematic regional variation. That is, the estimates are adjusted to remove the effect of random variation. Second stage estimates were calculated by shrinking the crude relative risks towards the mean value $(R R=1)$ such that the amount of variation in the second stage estimates was equal to the estimated level of systematic regional variation in survival estimated by $V$. A linear shrinkage of the form $b_{r}^{*}=\theta b_{r}$ was used, where $b_{r}$ is the original (crude) estimate and $b_{r}^{*}$ the second stage estimate for region $r=1, \ldots$, $p$. Since $\operatorname{var}\left(b^{*}\right)=\theta^{2} \operatorname{var}(b)$ we have $\theta=\sqrt{ }\left(V / s_{b}^{2}\right)$, where $s_{b}^{2}$ is the sample variance of the ensemble of parameter estimates. If $V \leqslant 0$ then $b_{r}^{*}=0$ for all regions, otherwise $b_{r}^{*}=\theta b_{r}$ is the second stage estimate of $\beta_{r}$ and $\exp \left(b_{r}^{*}\right)$ is the second stage estimate of the relative risk.

STEP 3: POTENTIAL MORTALITY SAVINGS We can use $V$ to identify those cancer sites that exhibit systematic regional variation (the larger the value of $V$, the larger the systematic variation), but it is difficult to grasp the practical implications of a given value of $V$. By estimating the number of deaths "saved" by eliminating any regional variation in survival, we are able to present results with a more concrete interpretation. The number of cancer deaths (deaths attributable to cancer) in an interval is defined as the number of deaths in the interval minus the number of expected deaths according to mortality in a comparable group in the general population. We define the number of deaths "saved" by removing regional variation in survival as the reduction in the number of cancer deaths during the first 10 years of follow up (15 years for breast cancer). The longer time frame was used for breast cancer because a significant number of breast cancer deaths occur in the period 10-15 years after diagnosis. ${ }^{24}$

Applying equations (2) and (3) to the estimated parameters from the regression model, it is a simple matter to estimate the relative survival ratio and the predicted number of deaths due to cancer for any combination of age, sex, and follow up year. The number of cancer deaths across all regions in follow up interval $j$ (and given levels of age and sex) is then defined as the number of deaths in the interval minus the number of expected deaths according to mortality in a comparable group in the general population and is given by

$$
C_{j}=l_{j} p_{j}^{*}\left(1-r_{j}\right)
$$

where $r_{j}$ is the (model based) interval specific annual relative survival ratio (for given levels of age and sex), $l_{j}$ is the number alive at the start of follow up interval $j$, and $p_{j}^{*}$ is the expected survival probability, taken from age/ sex specific population life tables.

The number of cancer deaths was calculated for each follow up year and summed across the first 10 follow up years (15 for breast cancer) to obtain an estimate of the total number of cancer deaths for each country, age group, and sex. The number of cancer deaths was then recalculated under the assumption that any existing systematic regional variations were eliminated and relative survival in all regions was equivalent to what would be expected if everyone received the same level of effective care. The percentage difference between these two quantities is an estimate of the percentage of cancer deaths potentially "savable" by removing systematic regional variation in survival. A percentage equal to $0.0 \%$ indicates that there was no evidence of systematic regional variation in survival so no deaths can be saved by eliminating such variation. A percentage of, say, $1.6 \%$ indicates that eliminating regional variation in survival would save an estimated $1.6 \%$ of cancer deaths. In calculating these percentages, it was assumed that everyone could be followed up for at least 10 years (15 years for breast cancer) in order to eliminate any potential bias due to differential withdrawal patterns. 
The excess mortality under the assumption that everyone receives the same level of effective care (no regional variation in survival) is given by

$$
v_{\text {risj }}^{\text {new }}= \begin{cases}\operatorname{RR}_{r}^{\text {new }} v_{r i j j} & \text { for } j=1, \ldots, 5 \\ v_{\tilde{r} i j j} & \text { for } j>5\end{cases}
$$

where $v_{\tilde{r} \text { is }}$ is the model based estimate of the excess cancer mortality for age $i, \operatorname{sex} s$, and follow up interval $j$ averaged over all regions and $R_{R}^{\text {new }}$ is the estimated relative risk reflecting the reduction in excess mortality we would observe if there was no regional variation in survival and everyone received the same level of effective care. Estimation of $v_{\text {risj }}$ is carried out by fitting a model without region. There was no evidence of regional variation in survival in follow up intervals $6-15$ so we assumed the excess mortality due to cancer would be unchanged after the fifth year of follow up.

The central issue in estimating the percentage of potentially savable deaths is how to estimate $\mathrm{RR}_{r}^{\text {new }}=\exp \left(\beta_{r}^{\text {new }}\right)$. Simply assuming that every region will attain the same relative survival ratio as the best (or even second best) region is not appropriate since these estimates are subject to random variation. The estimate $V$ represents the estimated variance of the ensemble of region specific parameters (log relative risks). If we assume that this ensemble is a sample from a normal distribution, then $95 \%$ of the parameters should lie in the interval $(-1.96 \sqrt{ }[V], 1.96 \sqrt{ }[V])$ and hence $95 \%$ of the relative risks in the interval $(\exp (-1.96 \sqrt{ }[\mathrm{V}]), \exp (1.96 \sqrt{ }[V]))$. We used $\mathrm{RR}_{r}^{\text {new }}=\exp (-1.96 \sqrt{ }[V])$ as the relative risk estimate corresponding to effective treatment and assumed that all regions would obtain this level if regional variation in survival were eliminated.

The percentages of potentially savable cancer deaths were then applied to the $\mathrm{CiN} 1 b^{2}$ predictions of the number of cancer deaths for the period $2008-12$ in order to estimate the number of potentially savable deaths during 2008-12. This time period was used since incidence and mortality predictions have previously been made for it. ${ }^{12}$

In the $\mathrm{CiN} 1 \mathrm{~b}$ project, predictions of the numbers of cancer deaths for the period 2008-12 were adjusted for time trends in excess mortality where appropriate. If these trends are attributable to trends in the level of regional variation, our method for estimating the number of savable deaths may be erroneous, since the $\mathrm{CiN} 1 \mathrm{~b}$ prediction of the number of cancer deaths during 2008-12 has already been (at least partially) adjusted for changes in the level of regional variation. To investigate if this was a problem we instead predicted the number of cancer deaths during 2008-12 under the assumption that excess mortality rates remained unchanged over time while making use of previously predicted changes in incidence rates and population distribution. The results obtained were very similar for the two methods so we decided to use the $\mathrm{CiN} 1 \mathrm{~b}$ predictions for the number of deaths during 2008-12 since the results obtained from this method have a more straightforward interpretation and were more relevant to the $\mathrm{CiN}$ project.

\section{OCIAL CLASS VARIATION}

Cancer survival for many sites in Finland has been shown to depend on social class. ${ }^{25} 26$ Each case in the Finnish material contained a measure of social class, based on occupational class, obtained from the 1970 population census by record linkage using the unique personal identification number allocated since 1967 to all Finnish residents. The social class variable contained six levels as defined by Valkonen et al; ${ }^{27}$ namely professional and higher administration, lower administration, skilled workers, unskilled workers, farmers, and others. These social class categories were also used to construct the social class specific population life tables for Finland.

The aim of the social class analysis was to estimate the potential mortality savings if everyone had the same survival as the highest social class (professional and higher administration). The analysis proceeded identically to the regional analysis up to the stage where equation (5) was applied to estimate the excess cancer mortality under the assumption that every region received the same level of effective care (no regional variation in survival). The next step in the social class analysis was more straightforward, we re-estimated the number of deaths under the assumption that everyone had the same relative survival as the highest social class and estimated the potential savings in deaths under this assumption.

\section{Results}

\section{EXPECTED MORTALITY}

There were small differences in the estimated relative survival ratios (RSRs) calculated using the three different baseline mortality tables (nationwide, region specific, and social class specific) for calculating expected mortality. These differences were not large enough to cause concern about using nationwide life tables for calculating expected survival. For example, breast cancer is more common in the upper social classes so we would expect the estimated RSR for breast cancer using the social class life table to be less than the RSR estimated using the general life table. The five year relative survival ratio for all 14216 breast cancer cases in Finland was 0.744 using the region specific life table, 0.743 using the social class specific life table, and 0.745 using the general population life table. The corresponding RSRs for the 1439 cases diagnosed in the highest social class were $0.797,0.786$, and 0.797 , respectively. These differences due to using different population life tables were minor when compared to the differences in RSRs between the regions or between the social classes. Relative survival ratios for all breast cancer cases (not adjusted for age and sex) ranged from 0.70 to 0.80 across the social classes and from 0.69 to 0.81 across the regions. We therefore had no serious concerns in using 


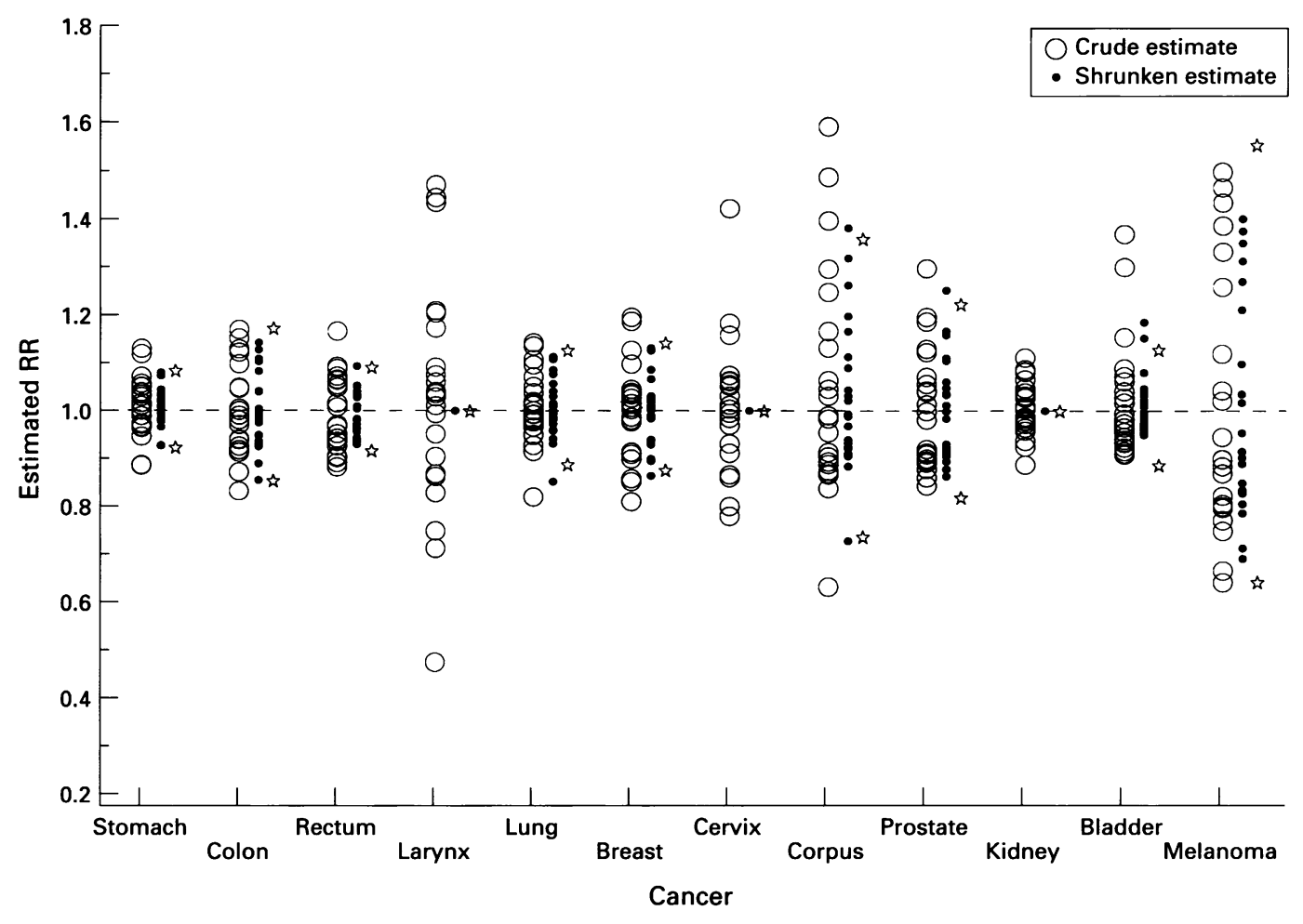

Figure 2: Crude and shrunken region specific estimates of the relative risk of excess mortality due to cancer in relation to site for 24 regions in Sweden, 1977-91. Estimates are from the regression model controlling for age, sex, follow up, and an age* follow up interaction. (Values of $\exp (-1.96 \sqrt{ }[V])$ and $\exp (1.96 \sqrt{ }[V])$ are represented by *.)

the general population life tables to estimate relative survival for regions and social class groups within each country.

\section{REGIONAL VARIATION}

Detailed results for Sweden are presented in figure 2 and table 3 . The open circles $(O)$ in figure 2 represent the crude estimates of the relative risks of excess mortality due to cancer and are identical to those presented in figure 1. The closed circles (O) represent the second stage estimates of the relative risks, shrunken such that the amount of variation in these estimates is equal to the estimated level of systematic regional variation in survival estimated by $V$. The estimated numbers of saved deaths are calculated under the assumption that on removing systematic regional variation in survival, all regions will have the same

Table 3 Significance of the region term in the regression model, variation in the crude estimate $\left(s_{b}^{2}\right)$, estimated $V$, total cancer deaths (CiN $1 \mathrm{~b}$ prediction ${ }^{2}$ ), and number and percentage of potentially savable cancer deaths in Sweden, 2008-12 due to removing regional variation in survival

\begin{tabular}{llrrrrr}
\hline Site & $\mathrm{p} \dagger$ & $s_{b}^{2}$ & $V$ & $\begin{array}{l}\text { Total } \\
\text { deaths }\end{array}$ & $\begin{array}{l}\text { Savable } \\
\text { deaths }\end{array}$ & $\%$ \\
\hline Melanoma & 0.00 & 731 & 507 & 2317 & 408 & 17.6 \\
Prostate & 0.00 & 141 & 105 & 12449 & 390 & 3.1 \\
Colon & 0.00 & 91 & 66 & 7699 & 382 & 5.0 \\
Breast & 0.00 & 96 & 46 & 10809 & 284 & 2.6 \\
Lung, trachea & 0.00 & 57 & 37 & 16576 & 249 & 1.5 \\
Urinary bladder & 0.02 & 130 & 38 & 4457 & 149 & 3.3 \\
Rectum & 0.03 & 59 & 20 & 5053 & 127 & 2.5 \\
Stomach & 0.00 & 28 & 17 & 5385 & 74 & 1.4 \\
Corpus uteri & 0.00 & 504 & 244 & 1401 & 16 & 1.1 \\
Larynx & 0.19 & 610 & -486 & 479 & 0 & 0.0 \\
Cervix uteri & 0.60 & 166 & -42 & 795 & 0 & 0.0 \\
Kidney & 0.71 & 34 & -17 & 3980 & 0 & 0.0 \\
Total & & & & 71400 & 2079 & 2.9 \\
\hline
\end{tabular}

t $\mathrm{p}$ value is based on comparing the change in deviance on removing the region term from the model to a $\chi^{2}$ distribution with $23 \mathrm{df}$. (effective care) relative risk, equal to $R_{R}^{\text {new }}=$ $\exp (-1.96 \sqrt{ }[V])$, which is represented by the lower of the two stars in figure 2 .

There was no evidence of systematic regional variation in survival for cancers of the larynx, cervix, and kidney as indicated by a negative value of $V$. The second stage estimates of the relative risks for these sites are therefore all equal to one (fig 2). Cancer of the larynx initially appeared to exhibit the highest level of regional variation in survival, but this was shown to be consistent with (or even less than) what we would expect due to random variation.

Systematic regional variation in cancer survival existed in each of the countries studied, although the level of variation differed according to site. All countries contained at least one site where there was no evidence of systematic regional variation. There were only minor differences in the results for males and females. Eliminating regional variation in survival in all countries would save an estimated $5271(2.5 \%)$ cancer deaths in the 12 study sites during the five year period 2008-12 (table 4). The corresponding number of deaths was 1282 (2.1\%) for Denmark, 1185 (2.9\%) for Finland, $724(1.9 \%)$ for Norway, and $2079(2.9 \%)$ for Sweden.

The sites in which the highest number of deaths could be saved are prostate, colon, melanoma, and breast (table 4). The largest percentage savings could be achieved for melanoma (11\%) and cervix uteri $(6 \%)$. Although the total percentage of savable deaths was similar for each country, there were differences between the countries within each site. No one country, however, was systematically higher or lower than the others. The differences 
Table 4 Total cancer deaths (CiN 16 prediction ${ }^{2}$ ) and number and percentage of potentially savable cancer deaths during 2008-12 due to removing regional variation in survival (all countries)

\begin{tabular}{lrcr}
\hline Site & $\begin{array}{l}\text { Total } \\
\text { deaths }\end{array}$ & $\begin{array}{l}\text { Savable } \\
\text { deaths }\end{array}$ & $\%$ \\
\hline Prostate & 29510 & 961 & 3.3 \\
Colon & 24331 & 846 & 3.5 \\
Melanoma & 6132 & 709 & 11.6 \\
Breast & 32011 & 708 & 2.2 \\
Lung, trachea & 59285 & 535 & 0.9 \\
Rectum & 14450 & 483 & 3.3 \\
Urinary bladder & 12465 & 409 & 3.3 \\
Stomach & 15713 & 210 & 1.3 \\
Cervix uteri & 2255 & 137 & 6.1 \\
Corpus uteri & 3920 & 120 & 3.1 \\
Larynx & 1830 & 80 & 4.4 \\
Kidney & 11344 & 73 & 0.6 \\
Total & 213246 & 5271 & 2.5 \\
\hline
\end{tabular}

Table 5 Colon cancer: number (\%) of potentially savable cancer deaths during 2008-12 due to removing regional variation in survival

\begin{tabular}{llr}
\hline Country & \multicolumn{2}{l}{ Savable deaths } \\
\cline { 2 - 3 } & Males & \multicolumn{1}{c}{ Females } \\
\hline Denmark & $101(2.9)$ & $151(3.8)$ \\
Finland & $84(4.4)$ & $96(4.6)$ \\
Norway & $13(0.5)$ & $18(0.7)$ \\
Sweden & $166(4.4)$ & $217(5.5)$ \\
Total & $363(3.15)$ & $482(3.8)$ \\
\hline
\end{tabular}

Table 6 Number and percentage of potentially savable cancer deaths during 2008-12 obtained by eliminating regional and social class variation in cancer survival in Finland

\begin{tabular}{|c|c|c|c|c|}
\hline \multirow[t]{2}{*}{ Site } & \multicolumn{2}{|l|}{ Region } & \multicolumn{2}{|c|}{ Social class } \\
\hline & $\begin{array}{l}\text { Savable } \\
\text { deaths }\end{array}$ & $\%$ & $\begin{array}{l}\text { Savable } \\
\text { deaths }\end{array}$ & $\%$ \\
\hline Stomach & 88 & 2.0 & 167 & 3.9 \\
\hline Colon & 180 & 4.5 & 101 & 2.5 \\
\hline Rectum & 137 & 6.5 & 138 & 6.5 \\
\hline Larynx & 5 & 1.8 & 27 & 8.7 \\
\hline Lung, trachea & 71 & 0.6 & 0 & 0 \\
\hline Breast & 187 & 2.7 & 248 & 3.5 \\
\hline Cervix uteri & 16 & 9.1 & 21 & 11.6 \\
\hline Corpus uteri & 30 & 3.5 & 147 & 17.5 \\
\hline Prostate & 173 & 3.3 & 57 & 1.1 \\
\hline Kidney & 0 & 0.0 & 78 & 2.6 \\
\hline Urinary bladder & 65 & 4.3 & 137 & 9.0 \\
\hline Melanoma & 231 & 24.3 & 95 & 10.1 \\
\hline Total & 1185 & 2.9 & 1217 & 3.0 \\
\hline
\end{tabular}

between countries for colon cancer (table 5) are shown as an example. A complete set of results is available from the authors upon request.

\section{SOCIAL CLASS VARIATION}

Eliminating social class variation in Finland will save an estimated $2.9 \%$ of cancer deaths during 2008-12. The savings are similar to those obtainable by eliminating regional variation, with the exceptions being cancer of the corpus uteri and melanoma (table 6 ). We would expect the results to be somewhat similar since there is an association between social class and region of residence. For melanoma there are greater differences in survival between regions than can be ascribed to social class differences and for corpus uteri the opposite is true. This implies cancer control strategies are important for melanoma at the regional level, while cancer of the corpus uteri requires strategies aimed at lower social classes

\section{Discussion}

The study objective was to examine equity in the health system by estimating the magnitude of systematic regional and social class variation in cancer survival. The results indicate that systematic regional variation (and hence inequity) exists in each of the countries studied. Results have been presented primarily in terms of the number of cancer deaths which could be saved by removing such variation. It is unlikely that regional variation in survival will be eliminated in the near future such that all regions obtain the "effective care" relative survival ratio assumed in the calculations. This is not to say, however, that the assumed "effective care" relative survival ratio is an unreasonable goal, since it has already been achieved by some regions for every site. The estimated number of savable deaths provides a ready method of quantifying the amount of systematic variation, thereby enabling identification of those sites where cancer control programmes may be most effective.

Note that we are not concerned with highlighting individual regions with high or low survival, but rather in finding the magnitude of the systematic variation in survival across all regions. It is for this reason that individual regions have not been identified in figure 1 . It is possible to assess the statistical significance of the regional variation using the change in deviance when the region term is omitted from the regression model. ${ }^{23}$ The regional effect is almost always significant due to the large number of regions and the large sample size so we learn little from this test (table 3). The level of statistical significance is dependent on sample size so cannot be used to rank the sites in terms of the level of systematic regional variation, as can be done with the statistic $V$.

There was a weak association between the relative survival ratio and the amount of regional variation in the 12 sites-sites with high relative survival generally had higher levels of regional variation. We could not, however, find any systematic pattern to explain why the level of regional variation differed between countries within each site. It may have been thought that, within a given site, the country with the highest survival may have a higher level of systematic regional variation but this was not the case. There was no association between the level of survival and the level of regional variation for the four countries within each site.

Potential bias due to regional differences in lead time was minimised by calculating the number of saved deaths from life tables covering long follow up intervals (10 or 15 years). This long follow up will not, however, overcome potential problems due to differential over diagnosis. If a particular cancer were, for example, over diagnosed in the higher social classes, these social classes would exhibit a higher incidence due to the over diagnosis and superior survival due to increased lead time. ${ }^{28}$ It would not be possible to raise the survival of the entire population to this level of survival since the level is somewhat artificial. 
Cases were excluded from the analysis if the diagnosis was first made at autopsy or if information was available from DCO. DCO cases are not included in data maintained by the Swedish cancer registry so were excluded from all data in order to maintain comparability. The effect of DCO cases on survival is not well defined and requires an understanding of the mechanism by which these cases are generated. ${ }^{29}$ It is generally accepted that if the true diagnosis dates of all DCO cases were known, the survival time of these cases would be considerably less than that of the "nonDCO" cases. Pollock and Vickers ${ }^{30}$ showed this empirically by tracing the true diagnosis dates of DCO registered cases in south east England.

The percentage of $\mathrm{DCO}$ cases in a given region depends upon the effectiveness of diagnostic, detection, and reporting procedures and the effectiveness of retrospective follow up (also called follow back) at the cancer registry. Regions with ineffective diagnostic, reporting, and retrospective follow up systems will generally have a higher proportion of DCO and autopsy cases (which have zero survival time) and a correspondingly lower proportion of cases with low survival times. Survival rates for such regions will be overestimated (compared to survival rates for other regions) if DCO and autopsy cases are excluded but underestimated if these cases are included in the analysis. The main issue, therefore, is not whether DCO and autopsy cases are included or excluded from an analysis but whether these cases are evenly distributed across regions. Examination of the regional distribution of DCO and autopsy cases showed no evidence of regional heterogeneity. To test further the effect of excluding these cases, the data were analysed without exclusions (apart from those cases where region of residence was not known). The estimated level of regional variation did not depend on whether or not cases were excluded. There were differences in the DCO rates between the four countries (table 2) although this will not affect the results since the analysis was conducted separately for each country.

Neither stage of disease at diagnosis nor year of diagnosis were included in the regression model. Stage is, of course, an important predictor of survival for all cancers, but it is not, however, justifiable to adjust survival rates for stage if the aim is to study the effect of the health care system on cancer survival. ${ }^{22}$ If we assume that stage depends mainly on diagnostic delay, stage is not a confounding factor but rather an outcome variable of interest (i.e. a chain in the causal link).

Year of diagnosis is a predictor of survival for many cancers but the amount of regional variation in survival is not heavily dependent on year of diagnosis. We fitted models containing predictor variables for year of diagnosis (two levels) and a year of diagnosis by region interaction term to all 12 sites for Finland and Sweden. As expected, year of diagnosis was statistically significant for nine of the 12 sites for both countries. It was not statistically significant for cancers of the larynx, lung, cervix uteri (Sweden), and corpus uteri (Finland).
The year by region interaction was significant in four of the 12 sites for each country, namely cancers of the stomach, colon, prostate, and kidney for Finland and cancers of the larynx, lung, female breast, and prostate for Sweden. Including these additional terms in the model had little effect of the estimates of regional variation, even for the sites where the year by region interaction term was significant. We decided to report the results using models without year of diagnosis as we wanted to minimise the number of strata in order to estimate the regional differences as efficiently as possible.

It must be noted that although the lack of regional variation in survival for a given cancer site is a positive indicator, it does not mean that survival cannot be improved. It is quite possible for a given country to have the lowest amount of regional variation in survival compared to other countries yet have the worst survival, or vice versa. The presence of regional variation is an indicator of possible inequity, which points towards a potential for improvement in survival. The absence of regional variation does not necessarily preclude the possibility of improving survival, but it may be more effective to initially focus on those cancers where regional variation exists.

The Nordic countries offer unique opportunities for conducting this type of research due to the high quality of the cancer registry data and the existence of civil registration systems which enable linking of the cancer registry database to obtain individual patient level information on education, occupation, and social class. This makes the Nordic countries an ideal platform for developing and testing the current methodology. The Nordic countries are justifiably proud of their health care systems, including the access to and quality of care available to all inhabitants. One concern at the start of this study was that we would not find any regional variation and would not be able to test the methodology properly, but this has not been the case.

The ability to study regional and social class variation in survival will greatly facilitate the evaluation of cancer control. The results from this study enable the identification of sites where variation, and hence possible inequity exists. These sites should be further studied in an effort to understand the underlying causes of the variation, followed by cancer control programmes to reduce the variation. The methodology developed for use in this study can be applied to future data in order to study changes in the level of regional variation over time. This research was supported in part by the Swedish Cancer
Foundation and the Nordic Cancer Union CiN project. We are most grateful to the staff of the Nordic cancer registries for most grateful to the staff of the Nordic cancer registries for
supply of the data and comments on the methodology. D supply of the data and comments on the methodology. Dr
Mike Kenward contributed to the discussion of the statistical Mike Kenward contributed to the discussion of the statistical
methodology and wrote a GLIM macro used in the study methodology and wrote a GLIM macro used in the study.
We wish to thank the anonymous referees, whose comments We wish to thank the anonymous referees, whose comments
substantially improved the paper.

1 Engeland A, Haldorsen T, Tretli S, et al. Prediction of the years 2000 and 2010. A collaborative study of the five Nordic cancer registries. APMIS 1993;101(suppl 38):000-000.

2 Engeland A, Haldorsen T, Tretli S, et al. Prediction of cancer mortality in the Nordic countries up to the years 
2000 and 2010. A collaborative study of the five Nordic can registries. APMIS 1995;103(suppl 49):000-000.

3 Cain K, Diehr P. Testing the null hypothesis in small area analysis. Health Serv Res 1992;27:267-93.

4 Diehr P, Cain K, Connell F, Volinn E. What is too much variation? The null hypothesis in small-area analysis. Health Serv Res 1990;24:741-71.

5 Westerling R. Components of small area variation in death rates: a method applied to data from Sweden. $\mathcal{f}$ Epidemiol Community Health 1995;49:214-21.

6 Boyle P, Muir CS, Grundmann E, eds. Cancer mapping. Berlin: Springer-Verlag, 1989.

7 Walter SD, Birnie SE. Mapping mortality and morbidity patterns: an international comparison. Int $\mathcal{f}$ Epidemiol 1991;20:678-89.

8 Walter SD. The analysis of regional patterns in health data. I Distributional considerations. Am $\mathcal{F}$ Epidemiol 1992;136: 730-41.

9 Walter SD. The analysis of regional patterns in health data. II The power to detect environmental effects. $A m \mathcal{F}$ Epidemiol 1992;136:742-59

10 World Health Organization. Plan of action implementing the global strategy for health for all. WHO Technical Report. Geneva: World Health Organization, 1982.

11 Karjalainen S. Geographical variation in cancer patient survival in Finland: chance, confounding, or effect of treat ment. F Epidemiol Community Health 1990;44:210-14.

12 Karjalainen S, Pukkala E. Social class as a prognostic factor in breast cancer survival. Cancer 1990;66:819-26.

13 Mooney G. Equity in health care: confronting the confusion. Effective Health Care 1983;1:179-85.

14 Mooney G. What does equity in health mean? World Health Stat $Q$ 1987;40:296-303.

15 National Health Strategy. Enough to make you sick: How income and envimnment affect health. Research paper No income and environment affect health. Research

16 Hakama M, Karjalainen S, Hakulinen T. Outcome based equity in the treatment of colon cancer patients in Finland. equity in the treatment of colon cancer patients in Finland. International fourna

17 Karjalainen S. Equity and cancer patient survival. Tampere: University of Tampere, 1991, PhD Thesis. Acta Universitatis Tamperensis 1991;316Series A.

18 Buckley JD. Additive and multiplicative models for relative survival rates. Biometrics 1984;40:51-62.

19 Estève J, Benhamou E, Croasdale M, Raymond L. Relative survival and the estimation of net survival: elements for further discussion. Stat Med 1990;9:529-38.

20 Henson DE, Ries LA. The relative survival rate. Cancer 1995;76:1687-88.

21 Hakulinen T, Tenkanen L. Regression analysis of relative survival rates. Applied Statistics 1987;36:309-17.

22 Hakulinen T. A comparison of nationwide cancer survival statistics in Finland and Norway. World Health Stat $Q$ statistics in Fin

23 Dobson A. An introduction to generalized linear modelling. London: Chapman and Hall, 1990

24 Hakulinen T, Pukkala E, Hakama M, Lehtonen M, Saxén E Teppo L Survival of cancer patients in Finland in E, Teppo L. Survival of cancer patients in Finland in

25 Rimpela A, Pukkala E. Cancers of affluence: positive socia class gradient and rising incidence trend in some cance forms. Soc Sci Med 1987;24:601-6.

26 Auvinen A, Karjalainen S, Pukkala E. Social class and cancer patient survival in Finland. Am f Epidemiol 1995 142:1089-102.

27 Valkonen T, Martelin T, Notkola V, Savela S. Socio-economic mortality differences in Finland 1981-90. Helsinki: Statistics Finland, 1993.

28 Saxén E, Hakama M. Cancer illnesss in Finland, with a note on the effect of age adjustment and early diagnosis. Annales Medicinae Experimentalis et Biologiae Fenniae 1964; Annales Medicinae 2 : 1-28.

29 Berrino F, Estève J, Coleman M. Basic issues in estimating and comparing the survival of cancer patients. In: Berrino and comparing the survival of cancer patients. In: Berrino F, Sant M, Verdecchia A, Capocaccia R, Hakulinen T, Estève J, eds. Survival of cancer patients in Europe: The EUROCARE study. IARC Scientific Publications No. 132. Lyon: International Agency for Research on Cancer, 1995. survival of cases registered by "death certificate only" implications for national survival rates. $\mathrm{Br} \mathcal{F}$ Cancer 1994 70:1229-31.

31 Francis B, Green M, Payne C, eds. The GLIM system: generalized linear interactive modelling. Rele

\section{Appendix}

THE VARIABILITY INDEX $V$

Let $\boldsymbol{\beta}=\left[\beta_{1}, \beta_{2}, \ldots, \beta_{p}\right]$ represent the regional log relative risk of excess mortality corrected for age, sex, and follow up time for the $p$ regions and $\mathbf{b}=\left[b_{1}, b_{2}, \ldots, b_{p}\right]$ be an estimator of $\beta$ with fixed but unknown covariance matrix $\mathbf{W}$. Our aim is to use the regression model ${ }^{21}$ to estimate the amount of systematic variation in the fixed effects $\boldsymbol{\beta}$.
The estimated parameters are a realisation of $\mathbf{b}$, with estimated covariance matrix $\hat{\mathbf{W}}$. The same notation is used for the estimators and the estimates, namely, $b_{1}, b_{2}, \ldots, b_{p}$. Maximum likelihood estimation is used (using GLIM $^{31}$ ) so $\mathbf{b}$ is an asymptotically unbiased estimator of B.

We chose the parameter

$$
\sigma_{\beta}^{2}=\frac{1}{p-1} \sum_{r=1}^{p}\left(\beta_{r}-\bar{\beta}\right)^{2}
$$

where

$$
\bar{\beta}=\sum_{r=1}^{p} \beta_{r} / p
$$

as the measure of the systematic regional variation and it is this parameter we wish to estimate. In effect, we are assuming that the ensemble of parameters $\beta_{1}, \ldots, \beta_{p}$ has a prior distribution with variance $\sigma_{\beta}^{2}$ and we wish to estimate $\sigma_{\beta}^{2}$. No assumptions are made about the form of the prior distribution. For the current application we have forced the mean of the $\beta_{r}$ s to zero so that the parameters have a ready interpretation but this property is not required in general.

An unbiased estimator for $\sigma_{\beta}^{2}$ is given by $V=$ $s_{\mathrm{b}}^{2}-\bar{V}_{1}+\bar{V}_{2}$, where

$$
s_{b}^{2}=\frac{1}{p-1} \sum_{r=1}^{p}\left(b_{r}-\bar{b}\right)^{2}
$$

is the sample variance of the ensemble of estimates;

$$
\bar{V}_{1}=\frac{1}{p} \sum_{r=1}^{p} \operatorname{var}\left(b_{r}\right)
$$

is the average of the variances of the estimators; and

$$
\overline{\mathrm{V}}_{2}=\frac{2}{p(p-1)} \sum_{r>s}^{p} \operatorname{cov}\left(b_{r}, b_{s}\right)
$$

is the average of the covariances of the estimators.

We do not know $\bar{V}_{1}$ and $\bar{V}_{2}$ so these must be estimated from the data. $\bar{V}_{1}$ is estimated by the average variance of the parameter estimates $\left(\hat{V}_{1}\right)$ and $\hat{V}_{2}$ is estimated by the average covariance of the parameter estimates $\left(\hat{V}_{2}^{\prime}\right)$, which are estimated by GLIM using the estimated expected information matrix.

If there is no systematic regional variation in survival between the regions $\left(\sigma_{\beta}^{2}=0\right)$, then $E(V)=0$ and the estimate for $\sigma_{\beta}^{2}$ can be either negative or positive. If regional variation does exist $\left(\sigma_{\beta}^{2}>0\right)$, then $E(V)>0$ and the estimate is less likely to be negative. A negative estimate for $\sigma_{\beta}^{2}$ means that we have observed less variation than would be expected due to chance so we conclude that there is no evidence of systematic variation. 